\title{
SWI/SNF is required for transcriptional memory at the yeast $G A L$ gene cluster
}

\author{
Sharmistha Kundu, ${ }^{1,2}$ Peter J. Horn, ${ }^{2}$ and Craig L. Peterson ${ }^{2,3}$ \\ ${ }^{1}$ Interdisciplinary Graduate Program, University of Massachusetts Medical School, Worcester, Massachusetts 01605, USA; \\ ${ }^{2}$ Program in Molecular Medicine, University of Massachusetts Medical School, Worcester, Massachusetts 01605, USA
}

\begin{abstract}
Post-translational modification of nucleosomal histones has been suggested to contribute to epigenetic transcriptional memory. We describe a case of transcriptional memory in yeast where the rate of transcriptional induction of GAL1 is regulated by the prior expression state. This epigenetic state is inherited by daughter cells, but does not require the histone acetyltransferase, Gcn5p, the histone ubiquitinylating enzyme, Rad6p, or the histone methylases, Dot1p, Set1p, or Set2p. In contrast, we show that the ATP-dependent chromatin remodeling enzyme, SWI/SNF, is essential for transcriptional memory at GAL1. Genetic studies indicate that SWI/SNF controls transcriptional memory by antagonizing ISWI-like chromatin remodeling enzymes.
\end{abstract}

[Keywords: Transcription; GAL1; SWI/SNF; chromatin, epigenetics, ISWI]

Supplemental material is availabe at http://www.genesdev.org.

Received October 30, 2006; revised version accepted February 28, 2007.

Specific patterns of gene expression are established during development, and these gene expression programs can be maintained through many cell divisions. The process of establishing and maintaining a transcriptional state that is heritable to progeny has been termed transcriptional memory. Within eukaryotic cells, chromatin structure plays a key role in establishing and maintaining ON/OFF states of gene expression. In its simplest state, chromatin is composed of long, linear arrays of nucleosomes that contain 147 base pairs (bp) of DNA wrapped about twice around an octamer of the core histones (two each of $\mathrm{H} 3, \mathrm{H} 4, \mathrm{H} 2 \mathrm{~A}$, and $\mathrm{H} 2 \mathrm{~B}$ ). Within cells, nucleosomal arrays are condensed into higher order structures, and the dynamic folding/unfolding of these structures is associated (and likely causative) with transcriptional activity.

Genetic and biochemical analyses of transcriptional regulatory mechanisms have led to the identification of two classes of highly conserved "chromatin remodeling/ modification" enzyme that regulate the dynamic state of chromatin (for reviews, see Becker and Horz 2002; Peterson and Laniel 2004). One class of chromatin remodeling/modification enzymes catalyzes the covalent attachment or removal of post-translational histone modifications (e.g., lysine acetylation, serine phosphorylation, lysine and arginine methylation, and lysine ubiquitylation). These histone marks can regulate the formation of higher order chromatin structures (e.g., H4-K16Ac) (Sho-

${ }^{3}$ Corresponding author.

E-MAIL Craig.Peterson@umassmed.edu; FAX (508) 856-5011.

Article is online at http://www.genesdev.org/cgi/doi/10.1101/gad.1506607.
gren-Knaak et al. 2006), or they can serve as the nucleating event for binding of nonhistone proteins that establish active or inactive chromatin states. For example, methylation of H3-K9 provides a binding site for the HP1 protein, which nucleates formation of repressive, heterochromatic structures (Grewal and Elgin 2002). HP1 can interact with the H3-K9 methyltransferase, which suggests a means for how this chromatin structure can be re-established following DNA replication (Grewal and Elgin 2002). Likewise, methylation of histone $\mathrm{H} 3$ at $\mathrm{K} 4$ is associated with transcriptionally active loci in many eukaryotes, and it has been suggested that $\mathrm{H} 3-\mathrm{K} 4 \mathrm{me}$ could provide a memory of previous transcriptional activity (Ng et al. 2003).

In addition to histone modifying enzymes, a distinct class of chromatin remodeling/modification enzyme uses the free energy derived from ATP hydrolysis to enhance the accessibility of nucleosomal DNA or change the histone composition of nucleosomes (Becker and Horz 2002; Smith and Peterson 2005). This family can be subdivided into at least five groups based on their biochemical properties and overall sequence similarity of their ATPase subunits: (1) SWI/SNF, (2) ISWI, (3) Mi-2/ CHD, (4) Ino80/Swr1, and (5) Rad54 (Flaus et al. 2006). Whereas many members of the ISWI-like and $\mathrm{Mi}-2 /$ CHD-like subgroups appear dedicated to transcriptional repression pathways (Kehle et al. 1998; Fazzio et al. 2001; Unhavaithaya et al. 2002), most SWI/SNF-like enzymes play roles in the activation of transcription (Peterson and Workman 2000). Notably, the Drosophila SWI/SNF complex harbors the Brm ATPase, which is a member of the TrX family of gene products that function as 
"memory factors" to maintain the transcriptional active state of homeotic genes during embryonic development (Tamkun et al. 1992).

Here we find that transcriptional induction of the yeast GAL1 gene exhibits "memory" of the preceding transcriptional state. Specifically, the rate of transcriptional induction of a naïve gene is slower than for a GAL1 gene that was previously transcribed. This ability to reinduce $G A L 1$ with fast kinetics survives at least one round of DNA replication and mitosis, indicating that this memory phenomenon is epigenetically inherited. Previous studies have demonstrated that nucleosomes at the GAL1 locus are subject to a variety of histone modifications during transcription, but we find that none of these marks are required for memory. In contrast, we find that inactivation of the SWI/SNF remodeling enzyme eliminates transcriptional memory at GAL1, such that the rate of transcriptional induction is nearly identical between a naïve gene and a GAL1 gene that had been previously transcribed. Surprisingly, we find that inactivation of ISWI-based chromatin remodeling enzymes restores transcriptional memory in a swi/snf mutant, suggesting that SWI/SNF prevents ISWI-based enzymes from erasing the memory of a previous round of transcription.

\section{Results}

\section{Transcriptional memory at GAL1 gene is heritable}

GAL1, which encodes the enzyme galactokinase, can be transcriptionally induced by $\sim 1000$-fold when yeast cells are grown in media containing galactose. Addition of glucose leads to rapid and efficient repression of GAL1 by multiple mechanisms, including a decrease in levels of the Gal4p activator and the galactose permease (Gal2p), and by activating several glucose repressor proteins that act at the GAL1 promoter (Johnston et al. 1994; Carlson 1998). Further, in neutral carbon sources such as raffinose, glycerol, or lactate, GAL1 is maintained in a poised state due to the masking of the Gal4p activation domain by the Gal80p repressor. We were specifically interested in how the trans-acting glucose repressors function, and therefore, we investigated the reinduction of GAL1 following a short period of glucose repression (see Fig. 1A). Cells were first grown in raffinose media so that GAL1 was poised for activation. Upon addition of galactose to the growth medium, GAL1 transcription commenced and transcripts appeared by 20 min post-induction (Fig. 1A,B). However, accumulation of maximum levels of GAL1 transcripts required $>1 \mathrm{~h}$ of growth in galactose media. Next, GAL1 expression was repressed by addition of $2 \%$ glucose and cells were grown for an additional hour. Surprisingly, when cells were washed into fresh media containing galactose, GAL1 transcription resumed very rapidly (Fig. 1A,B; Supplementary Fig. S2). Reinduction of GAL1 transcription peaked $<10 \mathrm{~min}$ after the addition of galactose (Fig. $1 \mathrm{~A}, \mathrm{~B})$. Thus, these results suggest that cells "remember" that GAL1 was previously transcribed, and conse- quently, they are poised to rapidly reinduce GAL1 transcription when galactose again becomes the dominant carbon source. Similar results were found for the reinduction of the GAL7 and GAL10 genes, indicating that this phenomenon is a general property of the $G A L$ gene cluster (data not shown).

We next tested whether the ability to reinduce GAL1 with rapid kinetics was a transient state or whether it could survive long-term growth in glucose media. Cells were grown overnight in raffinose media and then galactose was added for $60 \mathrm{~min}$ to induce GAL1 expression. Glucose was then added to repress GAL1, and at varying times after glucose addition, cell aliquots were transferred to galactose media and GAL1 reinduction kinetics were monitored. Figure 1C illustrates that cells grown for $2-4 \mathrm{~h}$ in glucose media retained the ability to rapidly reinduce GAL1 after subsequent addition of galactose (rapid induction defined as maximal expression at 20 min following galactose addition). In contrast, cells grown for $6-8 \mathrm{~h}$ in glucose reinduced GAL1 with the slower kinetics that mirrored induction kinetics of a naïve gene (Fig. 1, cf. lanes 10-15 in C and A). Since the yeast cell cycle is $\sim 2 \mathrm{~h}$ in glucose media, these results suggest that the ability to rapidly reinduce GAL1 might survive DNA replication and/or mitosis. To test this idea definitively, we performed an elutriation experiment (Fig. 1D). Cells were grown in galactose media until mid-log phase, and then cells were arrested at the G1/S transition of the cell cycle by treatment with $\alpha$ factor. Arrested cells were washed into glucose-containing media and then released from the cell cycle block and allowed to undergo one synchronous cell division in glucose media. Centrifugal elutriation was then performed in glucose media to isolate small, unbudded daughter cells from this culture. The daughter cell population was then transferred to galactose media and GAL1 reinduction kinetics were followed. The data shown in Figure $1 \mathrm{D}$ demonstrates that these daughter cells retained the ability to reinduce GAL1 with rapid kinetics (i.e., peak expression at $20 \mathrm{~min}$ ). These results indicate that memory of GAL1 gene transcription can be epigenetically inherited.

The transcriptional machinery is disassembled during glucose repression

One possibility is that rapid reinduction kinetics involves the persistent association of one or more components of the RNAP II transcription machinery with the GAL1 promoter during glucose repression. For instance, if TBP remains bound to the promoter during glucose repression, then reinduction might occur with faster kinetics. GAL1 transcription is activated by Gal4p, which binds to four sites within the $\mathrm{UAS}_{\mathrm{GAL}}$ between the GAL1 and GAL10 genes (Fig. 2A). When galactose is added to raffinose-grown cells, Gal80p-dependent inhibition of Gal4p is relieved, and Gal4p recruits a variety of transcription factors, including the SWI/SNF remodeling enzyme, the SAGA HAT complex, and the transcriptional mediator complex (Bhaumik and Green 2001; Lar- 
A

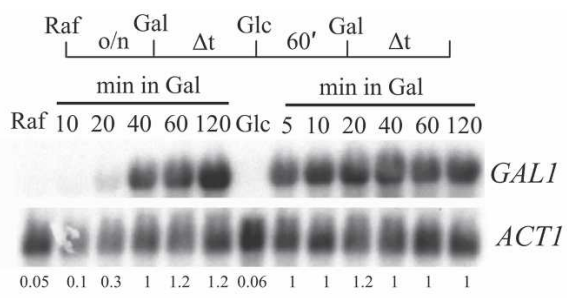

B

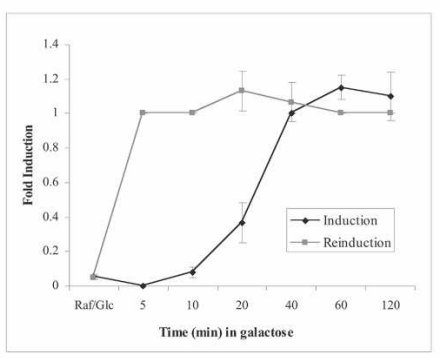

C

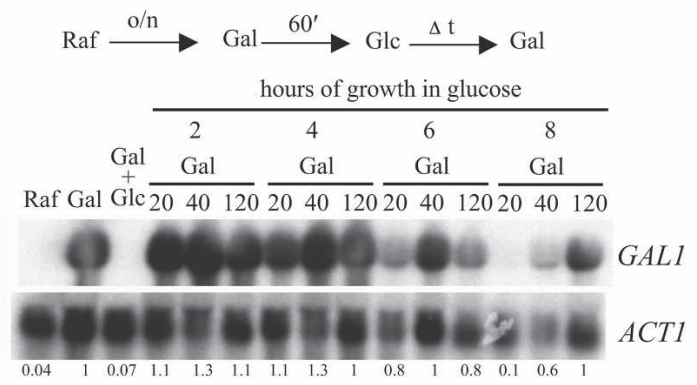

D
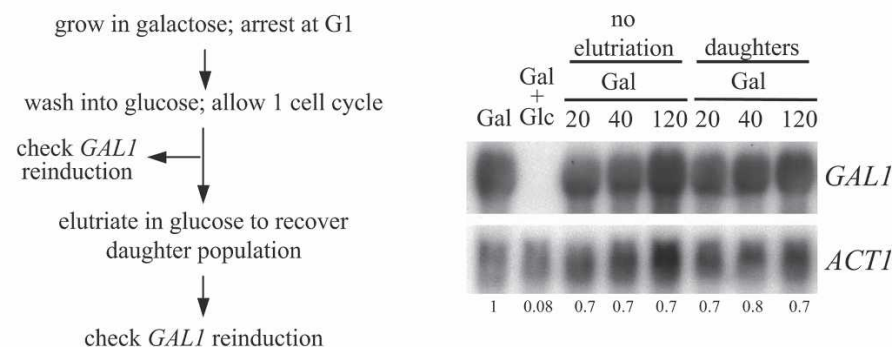

schan and Winston 2001; Lemieux and Gaudreau 2004; Dhasarathy and Kladde 2005). These events are followed by assembly of the preinitiation complex (PIC) and transcription of GAL1. We used chromatin immunoprecipitation (ChIP) to follow the recruitment of these factors to the GAL1 promoter during growth in galactose and during subsequent glucose repression (Fig. 2). As expected, robust levels of Gal4p were detected at $\mathrm{UAS}_{\mathrm{GAL}}$ when cells were grown in raffinose or galactose media. During glucose repression, Gal4p remained at high levels for the first $1 \mathrm{~h}$ in glucose, and then levels decreased approximately twofold at extended times in glucose media (Fig. 2B, top). The lower level of Gal4p was also observed in long-term glucose grown cultures and likely reflects partial occupancy of the multiple Gal4p-binding sites (Ren et al. 2000). These ChIP signals are specific to Gal4p since a gal4 $\Delta$ strain showed no enrichment of Gal4p at the $\mathrm{UAS}_{\mathrm{GAL}}$ (Fig. 2B, bottom). We also found that TBP, Mediator, RNA Polymerase II, SWI/SNF, and SAGA were recruited to the GAL1 promoter following galac-
Figure 1. Transcriptional memory at the GAL1 gene. (A) Northern analysis of GAL1 RNA levels. Schematic at top depicts regimen of growth in different carbon sources. (Raf) 2\% raffinose; (Gal) 2\% galactose; (Glc) 2\% glucose. Initial induction of GAL1 occurs with slower kinetics than when GAL1 is reinduced following glucose repression. $(B)$ Graph comparing kinetics of GAL1 induction and reinduction, averaged over three experiments performed as described in $A$. Error bars represent the standard deviation at each point. Slightly different time points were taken in different experiments, so in these cases no error bars are shown. (C) Cells were grown overnight in raffinose prior to addition of galactose. After $1 \mathrm{~h}$, cells were transferred to glucose media. At indicated times, an aliquot of cells was washed into fresh galactose media and GAL1 reinduction was observed. The "memory" state is maintained through at least $4 \mathrm{~h}$ of repression. (D) Glucose-grown daughter cells retain transcriptional memory. Cells were grown overnight in galactose media and then arrested at the G1/S boundary with $\alpha$ factor (lane labeled "Gal"). Arrested cells were then released from $\alpha$ factor into glucose medium to repress GAL1 and simultaneously undergo one synchronous division (lane labeled "Gal + Glc"). An aliquot of these cells was washed into galactose media to monitor reinduction kinetics (lanes labeled "no elutriation"). The remainder of the cells were elutriated to isolate daughter cells that had undergone mitosis in glucose media. Daughter cells were washed into galactose media to follow kinetics of GAL1 reinduction. The bottom panel represents an ACT1 loading control for total RNA levels. The numbers indicate fold induction of GAL1 transcripts normalized to ACT1 transcripts, with the maximally induced state set to a value of 1 . tose addition, but unlike Gal4p, none of these factors could be detected at GAL1 after $1 \mathrm{~h}$ of transferring the cells to glucose-containing medium (Fig. 2C). Thus, components of the transcription machinery rapidly dissociate from the promoter soon after GAL1 transcription is repressed by glucose addition. Since the ability to rapidly reinduce GAL1 persists for $2-4 \mathrm{~h}$ in glucose, these results suggest that this phenomenon is not due simply to persistent association of transcription factors with the promoter.

The kinetics of RNA Polymerase II recruitment to the GAL1 promoter was also analyzed throughout an induction/reinduction time course (Fig. 2D). In the initial round of GAL1 induction, high levels of RNAPII was detected at the GAL1 promoter within 20-40 min (Fig. 2D), consistent with the appearance of GAL1 mRNA (Fig. 1A). Strikingly, RNAPII was recruited much faster during reinduction of GAL1, with significant amounts of RNAPII detected 5 min after galactose addition. Similar results were found for recruitment of TBP /data not 
A

B

C

Figure 2. Rapid dissociation of the transcription machinery during glucose repression. $(A)$ Schematic representation of the GAL1-10 regulatory region. $\mathrm{UAS}_{\mathrm{GAL}}$ marks the Gal4p-binding sites. $\mathrm{URS}_{\mathrm{GAL}}$ contains the binding sites for the glucose repressor, Miglp. Regions covered by primers for ChIP are shown as horizontal lines. TATA represents the TBP-binding sites and +1 represents the transcription start sites. (B) Gal4p ChIP in wild-type and gal4s strains. (C) ChIP for TBP, RNA Polymerase II, Mediator ( $\alpha$-Srb4-13myc), SWI/SNF ( $\alpha$ Snf6), and SAGA ( $\alpha$-Spt3-13myc). For all factors, appropriate strains were grown in raffinose media until midlog phase, and GAL1 was induced for $1 \mathrm{~h}$ by adding galactose. Cells were then washed into media with glucose to repress GAL1 transcription for the indicated times. 3' GAL1 ORF, telomere (Chr VI-70 bp from the right end), and $A C T 1$ PCR primer sets were included as nonspecific controls. Numbers indicate ratio of percentage of immunoprecipitation values to corresponding nonspecific control. (D) RNA Polymerase II ChIP in wild-type strain grown for the reinduction regimen shown above the panel. Right panel shows quantitation of left panel.

shown). Thus, these results indicate that this phenomenon of GAL1 transcriptional memory occurs at the level of transcription initiation.

\section{Histone modifications are not required for transcriptional memory}

Post-translational modification of the core histones is an ideal candidate for an epigenetic mechanism that could contribute to the rapid GAL1 reinduction kinetics. Histone lysine methylation is particularly attractive, as this mark has a much longer half-life as compared with lysine acetylation (Katan-Khaykovich and Struhl 2005). Indeed, the transcription-associated methylation of histone $\mathrm{H} 3-\mathrm{K} 4$ (H3-K4me) has been hypothesized as a possible agent for memory of recent transcriptional activity ( $\mathrm{Ng}$ et al. 2003). We tested whether Setlp, which is responsible for H3-K4 methylation (Briggs et al. 2001; Roguev et al. 2001), or Set2p and Dot1p, which methylate H3-K36 (Strahl et al. 2002) and H3-K79 (Feng et al. 2002; Lacoste et al. 2002; Ng et al. 2002a; van Leeuwen et al. 2002), respectively, are required for transcriptional
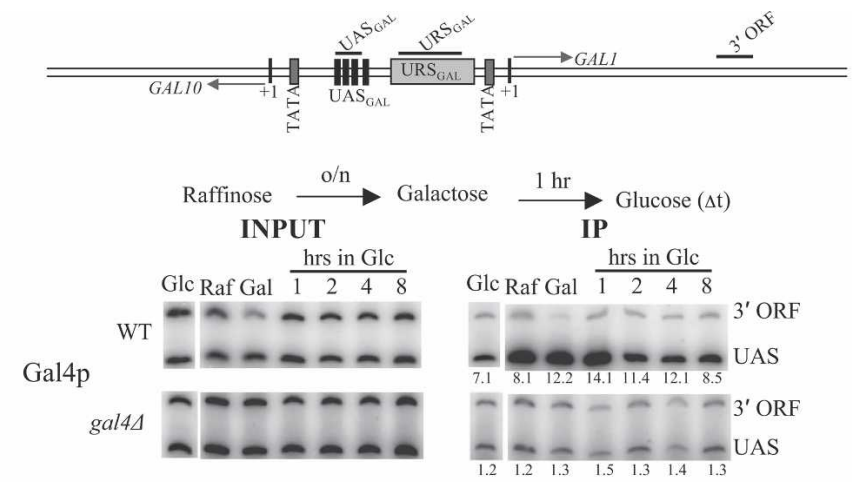

INPUT

IP
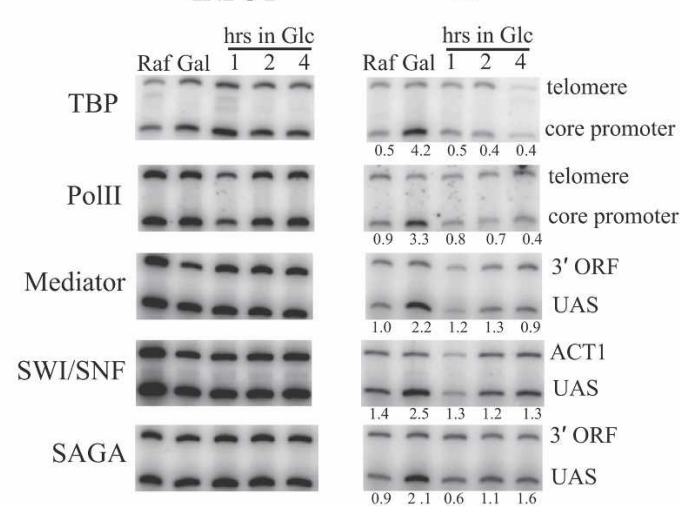

$-\underset{0.9}{2}-\frac{7}{0.6} \underset{0.1}{-} \underset{1.6}{-}$ UAS

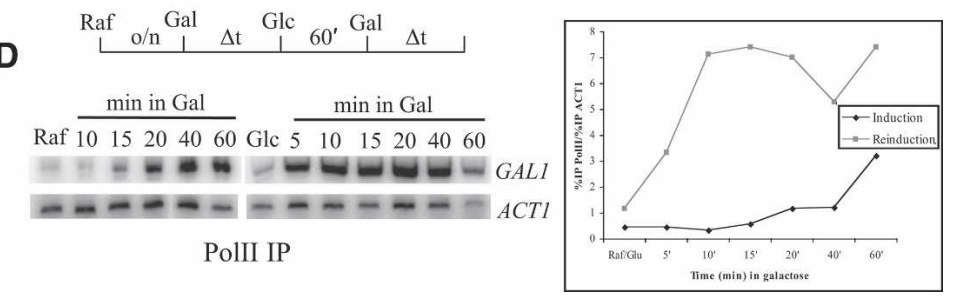

memory at GAL1. Interestingly, none of these methyltransferases are required for the rapid kinetics of GAL1 reinduction (Fig. 3A). After reinducing GAL1 transcription, transcript levels peaked within $20 \mathrm{~min}$ in set $1 \Delta$, set $2 \Delta$, or dot $1 \Delta$ strains (Fig. 3A). We also tested the reinduction kinetics of a rad $6 \Delta$ strain, since Rad6p-dependent ubiquitinylation of histone H2B-K123 is required for H3 methylation (Dover et al. 2002; Ng et al. 2002b; Sun and Allis 2002). In this case as well, GAL1 reinduction was rapid (Fig. 3B). Finally, we monitored reinduction kinetics in a $g c n 5 \Delta$ strain to eliminate SAGA-dependent acetylation of histone $\mathrm{H} 3$. In this case as well, no effect was observed on GAL1 memory (Fig. 3C). Thus, epigenetic modifications of histones do not appear to be responsible for imparting transcriptional memory at the GAL1 locus.

\section{Transcriptional memory requires SWI/SNF}

After determining that chromatin remodeling via histone modifications might not be required for the faster reinduction kinetics of GAL1, we turned to the ATP- 
A

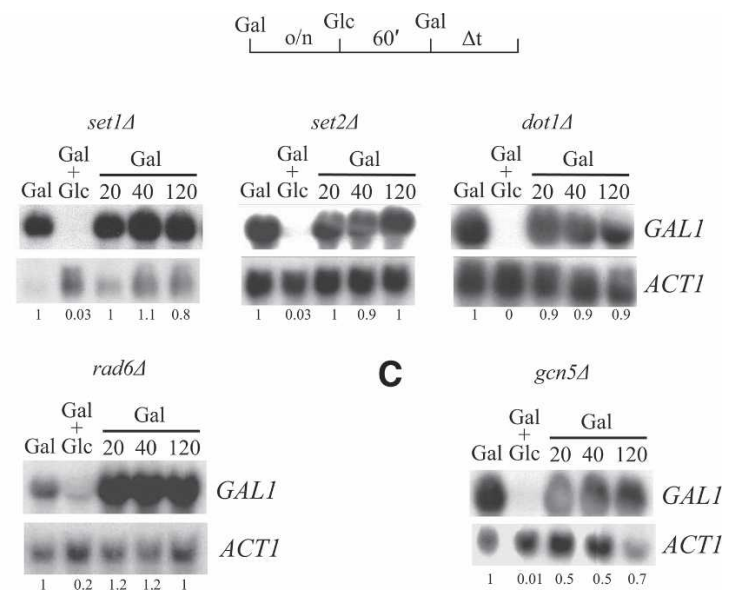

Figure 3. Histone-modifying enzymes are not essential for rapid GAL1 reinduction. Northern blot analyses are as in Figure 1. (A) Schematic at top illustrates growth media regimen. RNA was isolated from $\operatorname{set} 1 \Delta$, set $2 \Delta$, or $\operatorname{dot} 1 \Delta$ strains. (B) Identical analysis as in $A$, but with a rad6 6 strain. $(C)$ Identical analysis as in $A$, but with a $g c n 5 \Delta$ strain. All Northerns were subsequently probed for ACT1 as a loading control.

dependent chromatin remodeling enzymes. Previous studies have shown that SWI/SNF is recruited by the Gal4p activator to the GAL1 locus (Lemieux and Gaudreau 2004). Several studies have also shown that inactivation of SWI/SNF does not alter steady-state GAL1 expression (Burns and Peterson 1997; Lemieux and Gaudreau 2004). Likewise, we found that the kinetics of GAL1 induction in a swi2s strain are nearly equivalent to those of a wild-type strain (Fig. 4A). In contrast, the swi2 $\triangle$ strain was unable to rapidly reinduce GAL1 following a 1-h period of glucose repression. Indeed, the kinetics of induction and reinduction were nearly identical (Fig. 4A,B). This defect in reinduction kinetics was observed irrespective of whether raffinose-grown swi2s cells were switched to galactose for $2 \mathrm{~h}$ (Fig. 4A) or if they were grown overnight in galactose (Supplementary Fig. S2). Thus, it appears that inactivation of SWI/SNF eliminates the memory of previous GAL1 activation.

One possibility is that SWI/SNF is not involved in the mechanism of transcriptional memory but that SWI/ SNF is generally required to antagonize glucose repression. To test this idea, wild-type and swi2s cells were grown overnight in glucose, and then GAL1 induction kinetics were monitored after cells were transferred to galactose media. In both the wild-type and swi2s strains, GAL1 transcripts appeared by $4 \mathrm{~h}$ after galactose addition, demonstrating that SWI/SNF is not required to antagonize glucose repression at GAL1 during an initial round of transcriptional induction (Fig. 4C).

We next tested if the ATPase activity of SWI/SNF was required for transcriptional memory. GAL1 reinduction kinetics were monitored in a strain harboring the swi2K798A allele, which encodes an ATPase-defective version of the Swi2p catalytic subunit. GAL1 reinduction in the swi2K798A strain was much slower than the isogenic wild-type strain ( $40 \mathrm{~min}$ in swi2K798A vs. $\sim 5$ min in wild type), indicating that the enzymatic activity of SWI/SNF is essential for the ability to rapidly reinduce GAL1 transcription. We note that reinduction kinetics in the swi2K798A strain are reproducibly faster than the isogenic swi2 $\Delta$ strain, which may indicate an additional

A

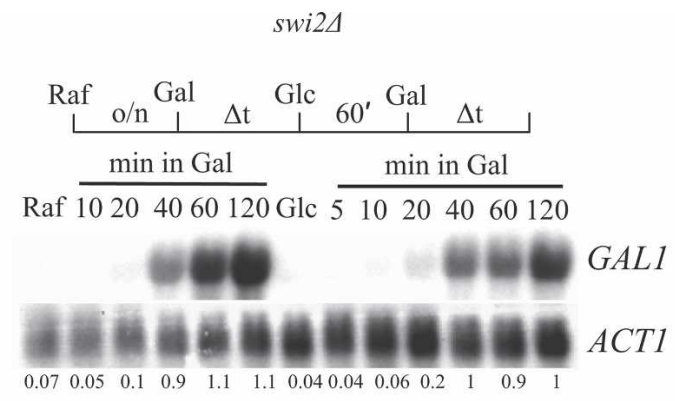

B

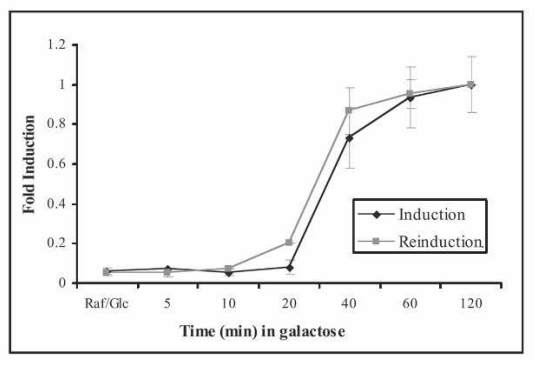

C

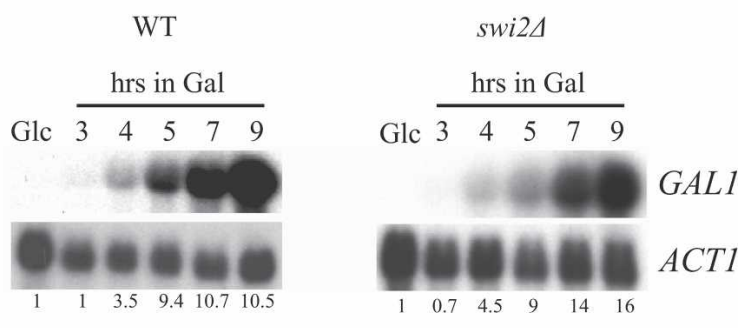

D

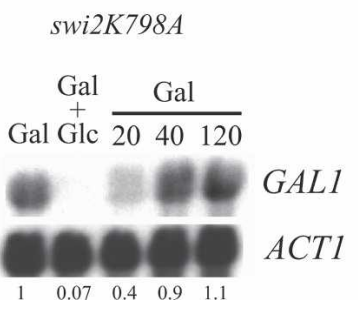

Figure 4. SWI/SNF is essential for GAL1 memory. (A) Northern analyses. Schematic at top illustrates the growth regimen for swi2 $\Delta$ cells. (B) Comparison of GAL1 induction and reinduction kinetics, averaged over three experiments performed as in $A$. Error bars represent the standard deviation at each point. Slightly different time points were taken in different experiments, so in these cases no error bars are shown. $(C)$ Isogenic wild-type and swi2s strains were grown overnight in glucose and then cells were transferred to galactose media. Both strains showed similar GAL1 induction kinetics after overcoming longterm glucose repression. $(D)$ An intact Swi2p ATPase domain is required for rapid GAL1 reinduction. Northern analysis of RNA isolated from cells harboring a swi2K798A allele, which inactivates the ATPase activity of SWI/SNF. 
ATP-independent role for SWI/SNF in GAL1 memory (Fig. 4, cf. D and A).

Loss of ISWI-like enzymes restores memory at GAL1 in the absence of SWI/SNF

Our data suggest that the initial round of GAL1 transcription establishes a heritable state that is poised for rapid reinduction. Previous studies have identified two changes in GAL1 chromatin structure that occur during transcriptional induction and that may contribute to this SWI/SNF-dependent memory state: (1) Two nucleosomes surrounding the GAL1 promoter are lost (Lohr and Lopez 1995), and (2) promoter-proximal nucleosomes are trimethylated at H3-K4 by the Setlp methyltransferase (Ng et al. 2003). The methylation of H3-K4 leads to the subsequent recruitment of an Isw1-containing, ATP-dependent chromatin remodeling enzyme (Santos-Rosa et al. 2003). When the transcriptionally active GAL1 gene is repressed by glucose, the promoter proximal nucleosomes are rapidly reassembled (Lohr 1984; Lohr and Lopez 1995), and high levels of $\mathrm{H} 3-\mathrm{K}_{4} \mathrm{me}_{3}$ persist (Ng et al. 2003; P.J. Horn and C.L. Peterson, unpubl.). One possibility is that SWI/SNF may influence the repositioning of promoter proximal nucleosomes such that subsequent PIC formation is favored. If this model is correct, then only small changes in nucleosome positioning are required, as Cavalli and Thoma (1993) have previously shown that the low resolution view $( \pm 50 \mathrm{bp})$ of GAL1 promoter nucleosomes is identical when samples are analyzed from cells grown long term in glucose or from galactose-induced cells that are treated with glucose for $<1 \mathrm{~h}$. Alternatively, the first round of GAL1 transcription may lead to a locus with a lower density of nucleosomes, even after subsequent glucose repression. Indeed, previous studies indicate that several hours of glucose repression is required before the entire GAL1 locus is restored to a regular nucleosomal array (Cavalli and Thoma 1993). SWI/SNF action may favor this chromatin state, whereas ISWI-like enzymes may promote the reassembly of a more regular nucleosomal array.

Although our data suggest that the SET1-dependent recruitment of Isw1p is not essential for GAL1 reinduction kinetics (Fig. 3A), we tested the possibility that a functional relationship exists between SWI/SNF and ISWI-like remodeling enzymes in transcriptional memory at GAL1. Budding yeast contain two distinct ISWIlike enzymes, Isw1 and Isw2 (Vary et al. 2003; Mellor and Morillon 2004), and each functions as the catalytic subunit of distinct multisubunit remodeling complexes. We created isogenic isw1 1 , isw2 2 , isw1 $1 \Delta$ swi2s, and isw2 $\Delta$ swi2 $\Delta$ strains and monitored GAL1 reinduction kinetics. Whereas the isw1 or isw2 single mutants had no effect on GAL1 induction or reinduction kinetics (Supplementary Fig. S1), we found that deletion of either ISW1 or ISW2 restored rapid reinduction kinetics in the swi2d strain (Fig. 5A,B). Furthermore, deletion of ISW2 allowed a swi2s strain to grow on galactose/anti-mycin solid media (data not shown). In contrast, inactivation of Set1p did not restore rapid GAL1 reinduction kinetics in
A
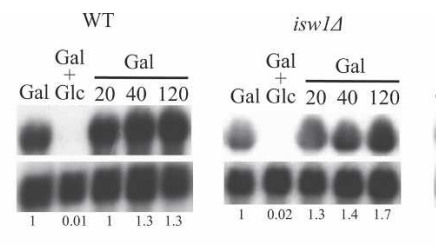

swi2 $\Delta$ iswlA

$\mathrm{Gal} \quad \mathrm{Gal}$

Gal Glc $\overline{20 \quad 40 \quad 120}$

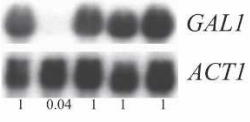

B
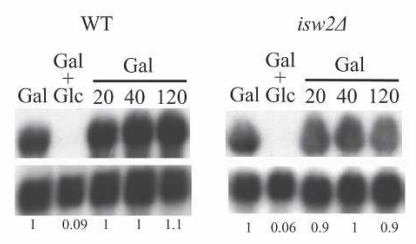

swi2 4 isw24

Gal Gal

Gal Gle $\stackrel{+}{2040120}$

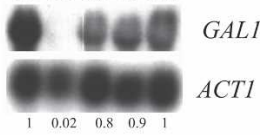

C
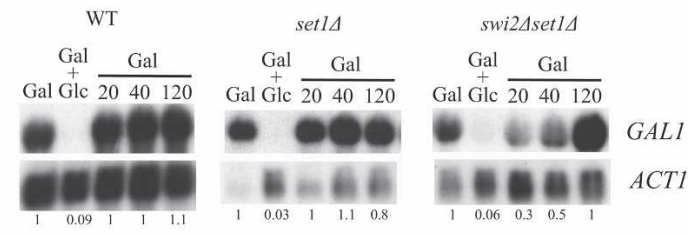

D

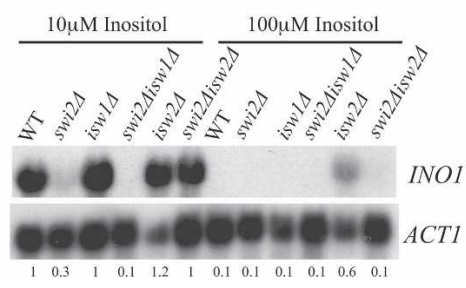

Figure 5. SWI/SNF antagonizes ISWI-like remodeling complexes. (A) Northern blot analysis for RNA isolated from wildtype (WT), isw1 $1 \Delta$, and isw1 $1 \Delta$ swi2 $\Delta$ strains. $(B)$ Northern analysis for RNA isolated from isw2 $2 \Delta$ and isw2 $\Delta$ swi2 $\Delta$ strains. $(C)$ Inactivation of Set $1 \mathrm{p}$ does not restore rapid reinduction kinetics in the absence of SWI/SNF. (D) Analysis of INO1 expression. The indicated isogenic strains were grown in minimal media containing high $(100 \mu \mathrm{M})$ or low $(10 \mu \mathrm{M})$ concentrations of myoinositol. Blots were reprobed for ACT1 as a control.

a swi2 mutant (Fig. 5C). Taken together, these results suggest that SWI/SNF controls memory of recent GAL1 transcription by antagonizing the repressive role of ISWI remodeling complexes. Such functional antagonism between SWI/SNF and ISWI-like enzymes is not unique to the GAL1 gene, as deletion of the ISW2 gene also alleviates the transcriptional requirement for SWI/SNF in the induction of the INO1 gene when cells are grown in low levels of inositol (Fig. 5D).

\section{Discussion}

How do SWI/SNF and ISWI-like complexes function at GAL1?

Our data suggest that the SWI/SNF that is recruited during the first round of GAL1 transcription potentiates the reinduction of GAL1 following a period of glucose repression. Furthermore, we found that this requirement 
for SWI/SNF can be bypassed by removal of the Isw1- or Isw2-based remodeling enzymes. Based on these results, we speculate that these two types of chromatin remodeling enzymes compete with each other to establish a GAL1 chromatin structure that is permissive for rapid reinduction of GAL1. As cells divide, this chromatin state can be inherited epigenetically but is eventually lost. In cells lacking SWI/SNF, the abundant Isw 1 and Isw2 enzymes establish an alternative, repressive chromatin state. In this situation, memory of the recent transcriptional activity is erased and slow reinduction kinetics result. Since SWI/SNF and ISWI-like enzymes tend to have opposing effects on the transcription of several yeast genes (including GAL1 and INO1), it is tempting to consider that such direct antagonism may be a more general phenomenon.

Recently, van Oudenaarden and colleagues (Acar et al. 2005) have described a distinct example of GAL1 transcriptional memory in which cells "remember" whether they were previously exposed to high or low concentrations of galactose. This particular memory phenomenon requires the GAL3 and GAL80 regulatory loops and likely involves the cytoplasmic inheritance of the positive regulator, Gal3p. Cytoplasmic inheritance of the Gal3p that is expressed during the first round of induction may contribute to the rapid induction kinetics that follows glucose repression. However, SWI/SNF plays a dominant role in transcriptional memory that is independent of Gal3p or the Gal2p permease since SWI/SNF does not affect GAL3 or GAL2 expression during the first round of galactose induction (Supplementary Fig. S3). Thus, transcriptional memory at GAL1 appears to involve a cytoplasmic mechanism that generally controls GAL1 expression levels, and a chromatin-based mechanism that specifically regulates the rate of transcriptional reinduction following transient glucose repression.

\section{Materials and methods}

Yeast strains, media, and growth conditions

Strains used in this study are isogenic derivatives of the S288c background. Genotypes are provided in Supplementary Table S1. Saccharomyces cerevisiae liquid cultures were grown at $30^{\circ} \mathrm{C}$ in YEP ( $1 \%$ yeast extract, $2 \%$ bacto-peptone) media supplemented with $2 \%$ glucose, $2 \%$ galactose, or $2 \%$ raffinose $+0.2 \%$ sucrose depending on whether GAL1 activation or repression was required. For reinduction studies, aliquots of glucose-grown cultures were centrifuged for $5 \mathrm{~min}$ at room temperature, and cell pellets were washed once with YEP, centrifuged for $5 \mathrm{~min}$, and resuspended in prewarmed YEPGal. To activate INO1 expression, cells were grown to mid-log phase in SD medium complete with amino acids. INO1 expression was repressed by adding $100 \mu \mathrm{M}$ myo-inositol. Deleted strains were made by a PCR-based method using kanamycin resistance cassette. Deletions were confirmed by PCR from genomic DNA with primers designed in the ORF of the individual deleted gene.

\section{Centrifugal elutriation}

Centrifugal elutriation of wild-type cells was performed as described (Johnston and Johnson 1997).
RNA isolation and analysis

Total RNA was isolated from yeast grown to logarithmic phase in appropriate media by hot phenol extraction method. Concentration of RNA was determined by measuring A260 after dissolving in diethyl pyrocarbonate-treated water. Ten micrograms (for GAL1 expression) or $25 \mu \mathrm{g}$ (for INO1 expression) of total RNA from each sample were electrophoresed on $1 \%$ formaldehyde agarose gels and Northern blotting was done. The housekeeping gene ACT1 was used as loading control. Radioactively labeled probes for hybridization were generated by PCR amplification of the complete GAL1, INO1, or ACT1 ORFs from genomic DNA.

\section{ChIP}

Mouse monoclonal antibody to the Gal4-DBD (RK5C1) was obtained from Santa Cruz Biotechnology. Anti-TBP and anti-Snf6 (for SWI/SNF) antibodies were kind gifts from M.R. Green (University of Massachusetts Medical School, Worcester, MA) and J. Reese (Pennsylvania State University). Mouse monoclonal antibody to RNA Polymerase II (CTD4H8) was obtained from Covance Research Products. SAGA (13-myc tagged Spt3) and Mediator (13-myc tagged Srb4) were immunoprecipitated with mouse monoclonal anti-Myc (9E10) antibody (Santa Cruz Biotechnology). ChIP assays were performed as described (Li et al. 2000). The immunoprecipitated DNA was amplified using quantitative PCR performed with $\alpha-{ }^{32} \mathrm{P}$-dCTP and then electrophoresed on $5 \%$ acrylamide gels. Reactions were visualized and quantified by PhosphorImager.

\section{$R T-P C R$}

Wild-type and swi2s cells were grown to mid-log phase in YEP medium with $2 \%$ glucose, $2 \%$ galactose, or $2 \%$ raffinose $+0.2 \%$ sucrose at $30^{\circ} \mathrm{C}$. Ten milliliters of cells were harvested and total RNA was extracted as described above. First-strand cDNA was synthesized using $2.5 \mu \mathrm{g}$ of RNA, SuperScript II RNase $\mathrm{H}^{-}$reverse transcriptase (Invitrogen), and 2 pmol each of GAL2, GAL3, or ACT1 downstream primers, following the manufacturer's instructions. Subsequently, ${ }^{32}$ P-labeled PCR was performed using $2 \mu \mathrm{L}$ of the first-strand cDNA reaction, and genespecific primer sets to determine the relative levels of $G A L 2$, $G A L 3$, and ACT1 mRNA for each strain. After 14 cycles (for ACT1) or 25 cycles (for GAL2 and GAL3) of amplification, PCR products were electrophoresed on $10 \%$ acrylamide gels.

\section{Acknowledgments}

We thank D. McCollum (UMMS) and N. Rhind (UMMS) for help with the elutriation studies, M.R. Green (UMMS) for antibodies, and F. Winston (Harvard Medical School) for yeast strains. This work was supported by an NIH grant to C.L.P. (GM49650) and a post-doctoral fellowship from the Charles King Trust to P.J.H.

\section{References}

Acar, M., Becskei, A., and van Oudenaarden, A. 2005. Enhancement of cellular memory by reducing stochastic transitions. Nature 435: 228-232.

Becker, P.B. and Horz, W. 2002. ATP-dependent nucleosome remodeling. Annu. Rev. Biochem. 71: 247-273.

Bhaumik, S.R. and Green, M.R. 2001. SAGA is an essential in vivo target of the yeast acidic activator Gal4p. Genes \& Dev. 15: 1935-1945.

Briggs, S.D., Bryk, M., Strahl, B.D., Cheung, W.L., Davie, J.K., 
Dent, S.Y., Winston, F., and Allis, C.D. 2001. Histone H3 lysine 4 methylation is mediated by Set1 and required for cell growth and rDNA silencing in Saccharomyces cerevisiae. Genes \& Dev. 15: 3286-3295.

Burns, L.G. and Peterson, C.L. 1997. The yeast SWI-SNF complex facilitates binding of a transcriptional activator to nucleosomal sites in vivo. Mol. Cell. Biol. 17: 4811-4819.

Carlson, M. 1998. Regulation of glucose utilization in yeast. Curr. Opin. Genet. Dev. 8: 560-564.

Cavalli, G. and Thoma, F. 1993. Chromatin transitions during activation and repression of galactose-regulated genes in yeast. Genes \& Dev. 12: 4603-4613.

Dhasarathy, A. and Kladde, M.P. 2005. Promoter occupancy is a major determinant of chromatin remodeling enzyme requirements. Mol. Cell. Biol. 25: 2698-2707.

Dover, J., Schneider, J., Tawiah-Boateng, M.A., Wood, A., Dean, K., Johnston, M., and Shilatifard, A. 2002. Methylation of histone $\mathrm{H} 3$ by COMPASS requires ubiquitination of histone H2B by Rad6. J. Biol. Chem. 277: 28368-28371.

Fazzio, T.G., Kooperberg, C., Goldmark, J.P., Neal, C., Basom, R., Delrow, J., and Tsukiyama, T. 2001. Widespread collaboration of Isw2 and Sin3-Rpd3 chromatin remodeling complexes in transcriptional repression. Mol. Cell. Biol. 21: 6450-6460.

Feng, Q., Wang, H., Ng, H.H., Erdjument-Bromage, H., Tempst, P., Struhl, K., and Zhang, Y. 2002. Methylation of H3-lysine 79 is mediated by a new family of HMTases without a SET domain. Curr. Biol. 12: 1052-1058.

Flaus, A., Martin, D.M., Barton, G.J., and Owen-Hughes, T. 2006. Identification of multiple distinct Snf2 subfamilies with conserved structural motifs. Nucleic Acids Res. 34: 2887-2905.

Grewal, S.I. and Elgin, S.C. 2002. Heterochromatin: New possibilities for the inheritance of structure. Curr. Opin. Genet. Dev. 12: 178-187.

Johnston, L.H. and Johnson, A.L. 1997. Elutriation of budding yeast. Methods Enzymol. 283: 342-350.

Johnston, M., Flick, J.S., and Pexton, T. 1994. Multiple mechanisms provide rapid and stringent glucose repression of GAL gene expression in Saccharomyces cerevisiae. Mol. Cell. Biol. 14: 3834-3841.

Katan-Khaykovich, Y. and Struhl, K. 2005. Heterochromatin formation involves changes in histone modifications over multiple cell generations. EMBO J. 24: 2138-2149.

Kehle, J., Beuchle, D., Treuheit, S., Christen, B., Kennison, J.A., Bienz, M., and Muller, J. 1998. dMi-2, a hunchback-interacting protein that functions in polycomb repression. Science 282: $1897-1900$.

Lacoste, N., Utley, R.T., Hunter, J.M., Poirier, G.G., and Cote, J. 2002. Disruptor of telomeric silencing-1 is a chromatin-specific histone H3 methyltransferase. J. Biol. Chem. 277: 30421-30424.

Larschan, E. and Winston, F. 2001. The S. cerevisiae SAGA complex functions in vivo as a coactivator for transcriptional activation by Gal4. Genes \& Dev. 15: 1946-1956.

Lemieux, K. and Gaudreau, L. 2004. Targeting of Swi/Snf to the yeast GAL1 UAS G requires the Mediator, TAF IIs, and RNA polymerase II. EMBO I. 23: 4040-4050.

Li, X.Y., Bhaumik, S.R., and Green, M.R. 2000. Distinct classes of yeast promoters revealed by differential TAF recruitment. Science 288: 1242-1244.

Lohr, D. 1984. Organization of the GAL1-GAL10 intergenic control region chromatin. Nucleic Acids Res. 12: 8457-8474.

Lohr, D. and Lopez, J. 1995. GAL4/GAL80-dependent nucleosome disruption/deposition on the upstream regions of the yeast GAL1-10 and GAL80 genes. J. Biol. Chem. 270: 27671-27678.
Mellor, J. and Morillon, A. 2004. ISWI complexes in Saccharomyces cerevisiae. Biochim. Biophys. Acta 1677: 100-112.

Ng, H.H., Feng, Q., Wang, H., Erdjument-Bromage, H., Tempst, P., Zhang, Y., and Struhl, K. 2002a. Lysine methylation within the globular domain of histone H3 by Dot1 is important for telomeric silencing and Sir protein association. Genes \& Dev. 16: 1518-1527.

Ng, H.H., Xu, R.M., Zhang, Y., and Struhl, K. 2002b. Ubiquitination of histone H2B by Rad6 is required for efficient Dot1mediated methylation of histone H3 lysine 79. J. Biol. Chem. 277: 34655-34657.

Ng, H.H., Robert, F., Young, R.A., and Struhl, K. 2003. Targeted recruitment of Set1 histone methylase by elongating Pol II provides a localized mark and memory of recent transcriptional activity. Mol. Cell 11: 709-719.

Peterson, C.L. and Laniel, M.A. 2004. Histones and histone modifications. Curr. Biol. 14: R546-R551.

Peterson, C.L. and Workman, J.L. 2000. Promoter targeting and chromatin remodeling by the SWI/SNF complex. Curr. Opin. Genet. Dev. 10: 187-192.

Ren, B., Robert, F., Wyrick, J.J., Aparicio, O., Jennings, E.G., Simon, I., Zeitlinger, J., Schreiber, J., Hannett, N., Kanin, E., et al. 2000. Genome-wide location and function of DNA binding proteins. Science 290: 2306-2309.

Roguev, A., Schaft, D., Shevchenko, A., Pijnappel, W.W., Wilm, M., Aasland, R., and Stewart, A.F. 2001. The Saccharomyces cerevisiae Set1 complex includes an Ash2 homologue and methylates histone 3 lysine 4. EMBO J. 20: 7137-7148.

Santos-Rosa, H., Schneider, R., Bernstein, B.E., Karabetsou, N., Morillon, A., Weise, C., Schreiber, S.L., Mellor, J., and Kouzarides, T. 2003. Methylation of histone H3 K4 mediates association of the Isw $1 p$ ATPase with chromatin. Mol. Cell 12: $1325-1332$.

Shogren-Knaak, M.A., Ishii, H., Sun, J.M., Pazin, M.J., Davie, J.R., and Peterson, C.L. 2006. Histone H4-K16 acetylation controls chromatin structure and protein interactions. Science 311: 844-847.

Smith, C.L. and Peterson, C.L. 2005. ATP-dependent chromatin remodeling. Curr. Top. Dev. Biol. 65: 115-148.

Strahl, B.D., Grant, P.A., Briggs, S.D., Sun, Z.W., Bone, J.R., Caldwell, J.A., Mollah, S., Cook, R.G., Shabanowitz, J., Hunt, D.F., et al. 2002. Set2 is a nucleosomal histone H3selective methyltransferase that mediates transcriptional repression. Mol. Cell. Biol. 22: 1298-1306.

Sun, Z.W. and Allis, C.D. 2002. Ubiquitination of histone H2B regulates $\mathrm{H} 3$ methylation and gene silencing in yeast. $\mathrm{Na}$ ture 418: 104-108.

Tamkun, J.W., Deuring, R., Scott, M.P., Kissinger, M., Pattatucci, A.M., Kaufman, T.C., and Kennison, J.A. 1992. brahma: A regulator of Drosophila homeotic genes structurally related to the yeast transcriptional activator SNF2/SWI2. Cell 68: $561-572$

Unhavaithaya, Y., Shin, T.H., Miliaras, N., Lee, J., Oyama, T., and Mello, C.C. 2002. MEP-1 and a homolog of the NURD complex component $\mathrm{Mi}-2$ act together to maintain germline-soma distinctions in C. elegans. Cell 111: 991-1002.

van Leeuwen, F., Gafken, P.R., and Gottschling, D.E. 2002. Dotlp modulates silencing in yeast by methylation of the nucleosome core. Cell 109: 745-756.

Vary Jr., J.C., Gangaraju, V.K., Qin, J., Landel, C.C., Kooperberg, C., Bartholomew, B., and Tsukiyama, T. 2003. Yeast Isw1p forms two separable complexes in vivo. Mol. Cell. Biol. 23: 80-91. 


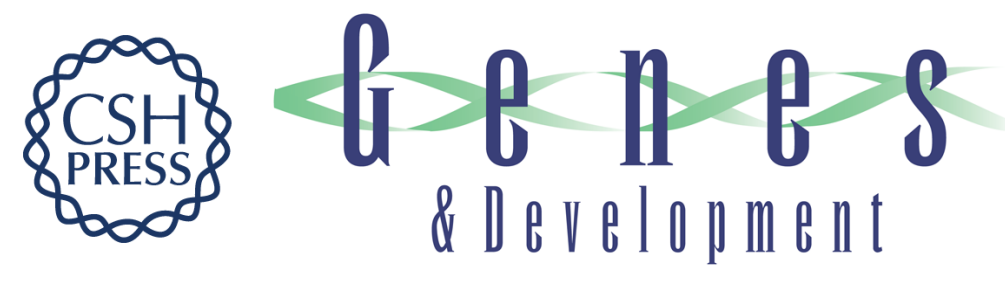

\section{SWI/SNF is required for transcriptional memory at the yeast GAL gene cluster}

Sharmistha Kundu, Peter J. Horn and Craig L. Peterson

Genes Dev. 2007, 21:

Access the most recent version at doi:10.1101/gad.1506607

\section{Supplemental http://genesdev.cshlp.org/content/suppl/2007/04/02/21.8.997.DC1 Material}

References This article cites 40 articles, 20 of which can be accessed free at: http://genesdev.cshlp.org/content/21/8/997.full.html\#ref-list-1

\section{License}

Email Alerting

Receive free email alerts when new articles cite this article - sign up in the box at the top Service

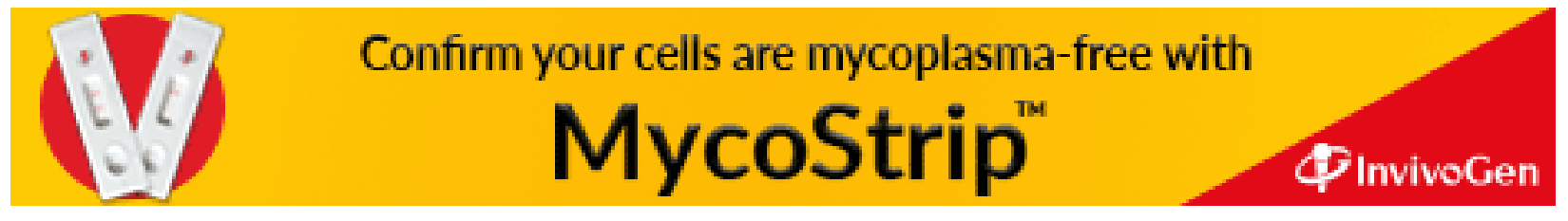

